\title{
México ante el COVID-19: Seguridad humana, gubernamentalidad y biopolítica
}

\author{
Edgar Ortiz-Arellano* \\ https://orcid.org/0000-0002-0907-7261
}

Recibido: 03 de mayo de 2020 • Aceptado: 21 de agosto de 2020

\section{Resumen}

Con la expansión acelerada de la enfermedad COVID-19, los diferentes gobiernos se enfrentan a condiciones que ponen a prueba sus capacidades para dar seguridad y protección a la vida de sus gobernados. Este artículo propone incorporar la noción de combate de esta epidemia desde la comprensión del funcionamiento de los dispositivos de la gubernamentalidad frente a estas amenazas de tipo biológico, de ahí que el objetivo del presente texto es dilucidar la problemática que ha provocado el COVID-19 en México desde las posturas de la biopolítica y la seguridad humana.

Palabras clave: Amenaza, políticas públicas, pandemia, gobierno mexicano, riesgos.

\footnotetext{
* Doctor en Gestión Estratégica y Políticas del Desarrollo, de la Universidad Anáhuac México, Campus Norte. Maestro en Administración de Negocios Internacionales de la Facultad de Contaduría y Administración (FCA), de la Universidad Nacional Autónoma de México (UNAM). Licenciado en Ciencia Política de la Universidad Autónoma Metropolitana-Iztapalapa (UAM-I). Catedrático en el posgrado de la FCA de la UNAM, así como en la Maestría en Ciencia Política del Centro de Estudios Superiores Navales (CESNAV), de la Secretaría de Marina-Armada de México. Correo: eortizarellano@comunidad.unam.mx
} 
Mexico versus COVID-19: Human Security, Governmentality and Biopolitics

\begin{abstract}
With the rapid spread of the COVID-19 disease, governments around the world face conditions that test their capabilities to provide security and protection to the lives of their governed. This paper proposes to incorporate the notion of combat of this epidemic from the understanding of the functioning of governmentality devices against these biological threats, hence the objective of this article is to elucidate the problems that COVID-19 has caused in Mexico from the positions of biopolitics and human security.
\end{abstract}

Key words: Threat, public policies, pandemic, Mexican government, risks.

\title{
Le Mexique face au Covid-19: sécurité humaine, gouvernementalité et biopolitique
}

\section{Résumé}

À cause de la propagation accélérée de la maladie Covid-19 les différents gouvernements doivent faire face aux conditions qui mettent à l'épreuve leurs capacités pour sécuriser et protéger la vie de leurs habitants. Cet article propose d'incorporer la notion de lutte contre cette pandémie du point de la vue de la compréhension du fonctionnement des dispositifs de la gouvernementalité face à ces menaces du type biologique. Ce texte a donc pour objectif d'élucider la problématique qu'a provoqué le Covid-19 au Mexique sous l'angle de la biopolitique et la sécurité humaine.

Mots-clés: Menace, politiques publiques, pandémie, gouvernement mexicain, risques. 


\section{Introducción}

En diciembre de 2019, a nivel mundial se reportó el brote de una nueva variedad de coronavirus, surgida en la provincia de Wuhan, China: SARS-CoV-2, la cual genera una enfermedad respiratoria sumamente infecciosa: COVID-19, que puede provocar la muerte a la persona contagiada. Enfrentar esta enfermedad ha sido un reto constante para los gobiernos de prácticamente todo el planeta, sobre todo en tres áreas: el sistema de salud, la economía y la seguridad nacional; estos sectores, se han visto afectados considerablemente por los efectos perniciosos de esta pandemia y por las medidas utilizadas para contrarrestarla.

Esta situación histórica que vive la humanidad, pone en el debate una serie de conceptos que en las últimas décadas del siglo XX fueron desarrolladas, en primera instancia, por Michel Foucault y, posteriormente, por pensadores como Giorgio Agamben, Paulo Virno, Judith Butler, Roberto Esposito, Antonio Negri, entre otros, con respecto de los cambios en la manera de gobernar que comenzaron a realizarse en el siglo XVIII. Las investigaciones que realizó Foucault describen y explican «el arte del gobernar» sobre los vivos, el cómo la gubernamentalidad incide sobre la vida humana para cuidar, desarrollar y volverla más productiva, así como consolidar su eficaz desempeño laboral y reproducción del capital, pero también para vigilarla en su comportamiento como población para predecir sus tendencias.

En este sentido, es necesario plantear, desde una postura crítica, cuál ha sido la actitud del gobierno mexicano para contener esta enfermedad. Este artículo propone incorporar la noción de combate de epidemias desde la dimensión de la seguridad con enfoque humano, para ello es necesario que se comprenda cómo funcionan los dispositivos de la gubernamentalidad frente a estas amenazas de tipo biológico, de ahí que el objetivo es dilucidar las problemáticas que ha provocado el COVID-19 en México desde las posturas de la biopolítica y el biopoder; para ello se apoya en el corpus teórico que Foucault desarrolló en torno a esta temática, al tomar como base la revisión de literatura, así como en el análisis de la situación de la epidemia en México entre los meses de febrero y fines de abril del 2020 (en la fase tres de la pandemia).

En su primer apartado, este ensayo explica el concepto de gubernamentalidad y las nociones del biopoder en torno de la seguridad; en el segundo punto se analizan las condiciones del caso mexicano y, posteriormente, se procede a las conclusiones.

\section{Gubernamentalidad y seguridad}

Michel Foucault observó que, especialmente en el siglo XVIII, hay un cambio en la manera de hacer gobierno, una transición del viejo poder soberano por una forma que está acompañada de una nueva función. «El derecho de soberanía es, entonces, el de hacer morir o dejar vivir. Y luego se instala el nuevo derecho: el de hacer vivir y dejar morir» ${ }^{1}$. Ahora a este poder le interesa la vida como fundamento del arte de

${ }^{1}$ Michel Foucault, Defender la Sociedad. Curso en el Collège de France (1975-1976) (Ciudad de México: Fondo de Cultura Económica, 2006), 218. 
gobernar, porque el punto de atracción será las poblaciones y una tecnología que aborda de manera científica los fenómenos que suceden en el seno de una sociedad; de hecho, para Foucault, este nuevo poder que se asentó en aquella época, sigue vigente en el orden global $^{2}$, por lo que tanto epidemias como endemias serán su tema de interés. «En ese momento, a fines del siglo XVIII, no se trata de esas epidemias sino de algo distinto: en líneas generales, lo que podríamos llamar las endemias, es decir, la forma, la naturaleza, la extensión, la duración, la intensidad de las enfermedades, reinantes en una población» ${ }^{3}$.

La lógica del gobierno y de sus dispositivos para intervenir sobre la vida consiste, en primera instancia, en la observación, análisis y predicción estadística de asuntos como las tasas de nacimientos, morbilidad y mortalidad, entre otras causas; así como determinar aquellas condiciones biológicas que impiden que la vida humana funcione de manera correcta ${ }^{4} \mathrm{y}$, con ello, determinar el conjunto de procedimientos de intervención gubernamental como son los servicios médicos, económicos y de seguridad, es decir, a las técnicas de gobierno, para las cuales «Foucault usó el término gubernamentalidad para describir esta intervención en la vida. Hacia la segunda mitad del siglo XVIII, entonces, estas técnicas de poder fueron acompañadas por una vigilancia disciplinaria dirigida a tomar el control de la vida y los procesos biológicos de la población para "regularizarlos", lo que Foucault denominó "hacer vivir y dejar morir" ${ }^{5}$ [traducción propia].

Para Foucault, el concepto tiene tres implicaciones, de las cuales, este artículo, pone énfasis en las dos primeras:

Entiendo el conjunto constituido por las instituciones, los procedimientos, análisis y reflexiones, los cálculos y las tácticas que permite ejercer esa forma bien específica, aunque muy compleja de poder que tiene por blanco principal la población, por forma mayor de saber la economía política y por instrumento técnico esencial los dispositivos de seguridad. Segundo por "gubernamentalidad" entiendo la tendencia, la línea de fuerza que, en todo Occidente, no dejó de conducir, y desde hace mucho, hacia la preeminencia del tipo de poder que podemos llamar "gobierno" sobre todos los demás 6 .

Esta forma de poder es el enraizamiento de las técnicas de gobierno en los asuntos públicos; pero, sobre todo, en las personas desde diversas perspectivas, especialmente la biológica. Todo esto desde una orientación racional y metódica, de ahí que: «El estudio de las formas de gubernamentalidad implica, entonces, el análisis de formas de racionalidad, de procedimientos técnicos, de formas de

${ }^{2}$ El orden mundial que se configuró en la década de 1990 pretende cimentarse en un sistema jurídico mundial de carácter biopolítico y disciplinar, este sistema Hardt y Negri lo llamaron Imperio. Al respecto, véase: Michael, Hardt y Antonio Negri, Imperio (Buenos Aires: Paidós, 2002).

${ }^{3}$ Foucault, Defender la Sociedad..., 221.

${ }^{4}$ Foucault, Defender la Sociedad..., 222-224.

5 Daria Davitti, «Biopolitical borders and the state of exception in the european migration "crisis"», European Journal of International Law 29, n. 4 (2018): 1177, acceso: 25 de abril, 2020, https://doi.org/10.1093/ejil/chy065

${ }^{6}$ Michel Foucault, Seguridad Territorio y Población. Curso en el Collège de France (19771978) (México: Fondo de Cultura Económica, 2006), 136. 
instrumentalización» ${ }^{7}$. Estas técnicas biopolíticas, aunadas a la expansión gubernativa y su racionalidad, pondrían énfasis en la seguridad que se debe dar a las poblaciones y, por lo tanto, al control que se ejerce sobre ellas para garantizar su sobrevivencia y desarrollo.

El gobierno, como núcleo rector del Estado, busca expandir su aparato y estructuras funcionales que le permitan mayor vigilancia y prevención de las emergencias, amenazas y riesgos que pudieran desestabilizar o destruir el orden establecido, así como a sus instituciones y población que gobierna, de ahí que construya andamiajes que permitan la protección de dichos elementos de la sociedad humana. La función del Estado hobbesiano, hasta la fecha, se centra en la preservación de la vida humana como una prioridad, pretensión que, en las condiciones actuales del mundo, es difícil de cumplir y acatar; sin embargo, el orden global intenta orientar las políticas hacia un enfoque de índole biopolítico, lo cual puede explicar el porqué del tránsito de la seguridad nacional, noción sumamente usada durante la Guerra Fría, hacia una propuesta de seguridad humana, impulsada desde 1994 por la Organización de las Naciones Unidas. «Tal enfoque implica proteger a la población de los riesgos y amenazas que pueden perjudicar sus aspiraciones y su calidad de vida, así como crear sistemas que faciliten el acceso de las personas a los derechos básicos de supervivencia, dignidad y trabajo decente» ${ }^{8}$.

El concepto de seguridad, que tiene como eje lo humano, es resultado de un proceso que deviene de las confrontaciones del siglo XX, lo cual, al terminar en la década de 1990, y con la amenaza de una conflagración de dimensiones planetarias, hizo que las políticas a nivel internacional se orientaran a construir una visión de índole biopolítica, «Desarrollada como una crítica a la priorización tradicional de la seguridad del Estado, la seguridad humana, tal como la define Commission on Human Security, tiene como objetivo la protección y la mejora del "núcleo vital de todas las vidas humanas"» ${ }^{9}$ [traducción propia].

La seguridad humana es un dispositivo orientado hacia la vida: «Como una tecnología centralizadora de la gobernanza internacional, la visión de la seguridad humana que comenzó a acelerarse a finales de la década de los noventa, implicó el aseguramiento biopolítico de poblaciones no aseguradas, mediante la unión de las prácticas, instituciones y redes de desarrollo sostenible existentes $\gg^{10}$. Los procedimientos (técnicas de gobierno) que la gubernamentalidad despliega son fundamentales, ya que esta nueva forma de poder transciende al Estado y se instala en la conducta de los individuos; es decir, una gubernamentalización total de la población, ahora el ciudadano toma consciencia de su propio cuidado y construye su

\footnotetext{
${ }^{7}$ Edgardo Castro, Diccionario Foucault. Temas, Conceptos y Autores (Argentina: Siglo XXI Editores, 2011), 177.

${ }^{8}$ Mirta Roses Periago, «Seguridad humana y salud pública: [editorial]», Rev. Panamericana de Salud Pública 31, n. ${ }^{\circ} 5$ (2012): 352, acceso: 26 de abril, 2020,

https://iris.paho.org/bitstream/handle/10665.2/9332/v31n5a00b_351-

4.pdf? sequence $=1 \&$ isAllowed $=\mathrm{y}$

${ }^{9}$ Hans-Martin Jaeger, «UN reform, biopolitics, and global governmentalit», International

Theory, Vol. II, Issue 1 (2010): 62, acceso: 01 de mayo, 2020, doi:10.1017/s1752971909990182

${ }^{10}$ Mark Duffield, «Seguridad humana: vincular desarrollo y seguridad en una era de terror», Relaciones Internacionales, $\mathrm{n}^{\circ} 43$ (2020): 28, acceso: 25 de abril, 2020, https://revistas.uam.es/index.php/relacionesinternacionales/article/view/relacionesinternaci onales2020.43.001/11880
} 
existencia en favor suyo (o así debería de ser). El individuo es responsable de aquello que lo mantiene vivo, sano y más en un contexto donde el Estado de bienestar es desmantelado para dar paso pleno a que el mercado lo controle todo. «El papel del Estado en la política pública neoliberal se caracteriza por una reducción de la política social a un mínimo, en especial en la distribución de beneficios sociales básicos para los pobres, fundamentalmente educación y salud, que son los servicios que garantizan la reproducción del "capital humano" ${ }^{11}$.

La gubernamentalización de la sociedad, por un lado, y la ampliación, así como la transición del concepto de seguridad nacional hacia seguridad humana, por otra parte, hacen que el campo de acción de las políticas en seguridad sean muy amplias, dejando a los poderes públicos la capacidad para decidir hasta dónde deben de intervenir, porque todo puede ser considerado una amenaza o riesgo; en este caso, una pandemia o, también, al usar los mismos argumentos, desestimarla y no considerarla como un tema inherente a la seguridad, es decir: «Los gobiernos pueden incluir bajo el título de amenaza cualquier aspecto que se les ocurra que afecte a sus intereses nacionales y geoestratégicos» ${ }^{12}$. Sin embargo, desde la perspectiva de la seguridad, el Estado debe proteger de cualquier tipo de amenaza a la población, sea este por ejemplo de tipo terrorista, desastre natural, pobreza generalizada o enfermedades como es el caso que aqueja ahora al mundo.

Para lograr esta protección a la que aspira el arte de gobernar, es necesario que los instrumentos de seguridad se ajusten a las tecnologías del biopoder, esto implica que son aparatos dinámicos, adaptados a la coyuntura y a las prácticas de intervención que, definidas por los hacedores de políticas, no surgen de manera espontánea. «Las prácticas de seguridad biopolítica no se articulan en un diseño de la naturaleza. Son logros contingentes que reflejan la realización parcial de diseños que buscan representar "naturalezas". En el proceso, hay deslizamientos, roturas, cambios y revisiones, para los cuales los operadores originales y las preocupaciones de la biopolítica ya no las consideran ${ }^{13}$ [traducción propia].

\section{México y COVID-19}

El Estado mexicano se rige por la Constitución Política de los Estados Unidos Mexicanos la cual, en su artículo 89, fracción VI, contempla que el presidente de la República es el responsable de preservar la seguridad nacional de país ${ }^{14}$; en esta misma norma constitucional, en su artículo 73, fracción XVI, se señala que:

\footnotetext{
${ }^{11}$ Ariadna Estévez, «La repolitización de los derechos humanos frente a la gubernamentalidad neoliberal del sufrimiento social: una lucha de contraconducta», en Ariadna, Estévez y Daniel Vázquez (coords), 9 Razones para (Des)confiar de las Luchas por los Derechos Humanos (Ciudad de México: Universidad Nacional Autónoma de México, Facultad Latinoamérica de Ciencias Sociales, Sede México, 2017), 184.

12 Nuria Hernández García, «La seguridad humana: del concepto al enfoque. Causas de la reducción de su uso como concepto», en Relaciones Internacionales, n. ${ }^{\circ} 43$ (2020): 46, acceso: 25 de abril, 2020, https://doi.org/10.15366/relacionesinternacionales2020.43.002

${ }^{13}$ Michael Dillon y Luis Lobo-Guerrero, «Biopolitics of Security in the 21st Century: An Introduction», Review of International Studies 34, n. ${ }^{\circ} 2$ (2008): 267, acceso: 30 de julio, 2020, doi: 10.1017/S0260210508008024

${ }^{14}$ Constitución Política de los Estados Unidos Mexicanos (México: Diario Oficial de la Federación, última reforma 06-03-2020): 88, acceso: 27 de abril, 2020, http://www.diputados.gob.mx/LeyesBiblio/pdf/1_060320.pdf
}

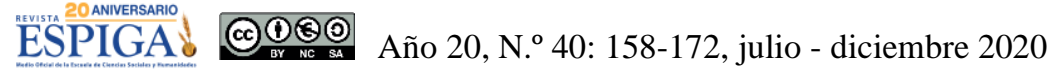


XVI. Para dictar leyes sobre nacionalidad, condición jurídica de los extranjeros, ciudadanía, naturalización, colonización, emigración e inmigración y salubridad general de la República.

1a. El Consejo de Salubridad General dependerá directamente del presidente de la República, sin intervención de ninguna Secretaría de Estado, y sus disposiciones generales serán obligatorias en el país.

2a. En caso de epidemias de carácter grave o peligro de invasión de enfermedades exóticas en el país, la Secretaría de Salud tendrá obligación de dictar inmediatamente las medidas preventivas indispensables, a reserva de ser después sancionadas por el presidente de la República.

3a. La autoridad sanitaria será ejecutiva y sus disposiciones serán obedecidas por las autoridades administrativas del País ${ }^{15}$.

El gobierno mexicano gira en torno del poder presidencial, esto como resultado de su devenir histórico y del proceso revolucionario que vivió entre 1910 y 1929, lo que orilló a que se constituyera un presidente fuerte con respecto a los otros poderes de la Unión (Legislativo y Judicial), así también, sobre los caudillos políticos y militares que dominaron el escenario político durante buena parte del siglo XIX y durante la Revolución mexicana. En la actualidad, el jefe del Ejecutivo concentra tanto a la administración pública centralizada como a los organismos descentralizados y empresas paraestatales, lo cual le da una presencia política muy importante de facto, que obliga a todos los actores políticos, económicos y sociales a converger en él, por lo que los temas de salud y control de enfermedades es un asunto que está constantemente en la agenda presidencial, más por las atribuciones constitucionales señaladas.

En caso de epidemias graves, son el Consejo de Salubridad General, el presidente de la República y la Secretaría de Salud, los encargados de encabezar las acciones para afrontar este tipo de amenazas a la población. Por otra parte, La Ley de Seguridad Nacional, en su artículo 3, fracción primera, menciona, con respecto de las tareas en su materia, que una de ellas es: «La protección de la nación mexicana frente a las amenazas y riesgos que enfrente nuestro país» ${ }^{16}$; sin embargo, en ningún apartado hay mención de las enfermedades o epidemias como amenazas o riesgos a la seguridad de la población o del Estado. La Ley General de Salud, en su artículo 181, indica que: «En caso de epidemia de carácter grave, peligro de invasión de enfermedades transmisibles, situaciones de emergencia o catástrofe que afecten al país, la Secretaría de Salud dictará inmediatamente las medidas indispensables para prevenir y combatir los daños a la salud, a reserva de que tales medidas sean después sancionadas por el presidente de la República ${ }^{17}$. Aquí, se observa cómo en el caso mexicano existe un andamiaje jurídico gubernamental de índole biopolítico en materia de salud, donde el saber médico tiene el control del combate a las epidemias

\footnotetext{
${ }^{15}$ Ibíd., 70.

${ }^{16}$ Ley de Seguridad Nacional (México: Diario Oficial de la Federación, últimas reformas 08-11-2019): 1, consulta: 27 de abril, 2020, http://www.diputados.gob.mx/LeyesBiblio/pdf/LSegNac_081119.pdf

${ }^{17}$ Ley General de Salud (México: Diario Oficial de la Federación, últimas reformas 24-012020): 81, acceso: 27 de abril, 2020, http://www.diputados.gob.mx/LeyesBiblio/pdf/142_240120.pdf

ESPIGA' @(1)(@) Año 20, N. ${ }^{\circ} 40: 158-172$, julio - diciembre 2020
} 
y no las áreas de seguridad nacional (o por lo menos de manera pública), estas últimas podrían aportar los análisis de inteligencia y análisis de la información que se generaban en todo el mundo y, con ello, prever la llegada del COVID-19 a México (vía personas procedentes de EE. UU., Europa y China) para así aminorar las posibilidades de contagio en el país. Pero también, a partir de los datos de morbilidad de la población y demás datos biológicos, los órganos de seguridad podrían haber desarrollado escenarios y estrategias para reducir el impacto de la tasa de mortalidad provocada por esta enfermedad.

El Estado mexicano, al igual que la mayoría de los Estados occidentales capitalistas, se configuró en un orden biopolítico en la tesitura planteada por Foucault, de ahí que el poder político de México pusiera énfasis en la protección sanitaria de la población a partir de la creación de instituciones hospitalarias y de seguridad popular, como lo son el Instituto Mexicano del Seguro Popular, Instituto de Seguridad y Servicios Sociales de los Trabajadores del Estado, Seguro Popular y, recientemente, el Instituto de Salud para el Bienestar, así como en el seguimiento estadístico de la población en cuanto a su comportamiento, en particular a través del Instituto Nacional de Estadística, Geografía e Información.

A lo largo de siglo $\mathrm{XX}$, el régimen creó un amplio andamiaje de instrumentos de seguridad y vigilancia sobre la población, que hasta la fecha siguen operando. Cabe señalar que estos dispositivos son tanto para salud y seguridad pública, como para seguridad nacional; todos ellos desarrollados por el Estado para la protección de la población, con el fin de hacerla vivir, para ejercer un gobierno sobre los vivos y evitar, por ejemplo, que la pandemia cause graves estragos en la vida de las personas, en este caso, por lo menos, a nivel normativo, pero no necesariamente en la realidad. Para el caso mexicano, la estrategia biopolítica en cuanto al COVID-19 ha consistido en dejar que la epidemia evolucione de manera natural y que el ciudadano sea el encargado, a partir del aislamiento y la higiene personal (Jornada Nacional de Sana Distancia), de contener esta enfermedad y, por otra parte, el Estado mexicano se ocupa de administrar los servicios hospitalarios históricamente deficientes (mencionados anteriormente) y que, ante la inevitabilidad de la pandemia, solo queda la opción de gestionar el saber médico lo mejor posible, así como la administración de la muerte de los pacientes. «En México hasta el día de hoy se han confirmado 408,449 casos y 45,361 defunciones por COVID-19» ${ }^{18}$. El 27 de febrero de 2020 se dio a conocer el paciente cero en México y, desde entonces y hasta la cuarta semana de abril, se llegó a la fase tres, donde ya no se puede dar seguimiento a la cadena de contagios, por lo que los enfermos se dan de manera masiva, lo cual ocasiona el colapso en el sistema de salud. En el caso de México (y como casi en todo el mundo), es en las zonas urbanas donde principalmente se ha dado el contagio, debido a que es el espacio donde hay mayor densidad poblacional. Este fenómeno Foucault lo detectó en las preocupaciones que existan con respecto a la relación epidemias-ciudad en el siglo XVIII ${ }^{19}$.

${ }^{18}$ Gobierno de México, Comunicado Técnico Diario COVID-19 México (Ciudad de México: Gobierno de México, 30/04/2020): 2, acceso: 30 de julio, 2020, https://www.gob.mx/cms/uploads/attachment/file/566650/Comunicado_Tecnico_Diario_C OVID-19_2020.07.29.pdf

${ }^{19}$ Michel Foucault, El Poder, una Bestia Magnífica (Ciudad de México: Siglo XXI Editores, 2013), 222-225.

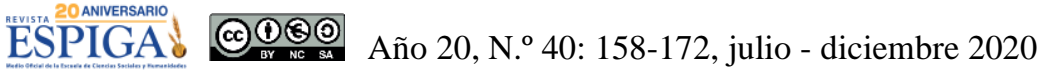


Ante esta nueva enfermedad, el Estado mexicano intentó utilizar la racionalidad gubernamental, realizó proyecciones estadísticas, generó estudios y brindó información diaria a la población ${ }^{20}$, entre otras medidas, para tratar de encauzar a la población hacia la gobernabilidad y, con estas acciones, tratar de evitar el pánico, revueltas populares, saqueos y el derrumbe económico. En este rubro, al principio de la expansión de la pandemia, en el país se decidió no detener la actividad económica $^{21}$, pero esta decisión, conforme creció el número de contagios, fue imposible de mantener. El no haber detenido la actividad económica se puede explicar si se considera que, en un orden social, donde el mercado es el agente económico más importante, las operaciones comerciales, los negocios, el flujo de mercancías, bienes y servicios no pueden parar, ya que al hacerlo provoca un efecto negativo en todos los ámbitos de la sociedad, en donde todos los sectores son dañados, pero aún más en aquellos que de por sí eran vulnerables antes de la pandemia y que, por el apremio de obtener recursos, arriesgan su integridad física por contagio de COVID-19, ya que en el sistema capitalista, no solo las técnicas biopolíticas y del cuidado del sí, ayudan a preservar la vida, es necesario ocupar un lugar en la cadena productiva del mercado para poder vivir.

El orden biopolítico es un espacio donde la acumulación y recopilación de información es vital para alimentar la supuesta racionalidad de las políticas que se implementan, más en situaciones de emergencia epidemiológica como la que se presenta ahora. «En este ámbito, la visibilidad de lo social se hace posible en la medida que se constituyen dispositivos de observación que son conformados por la elaboración de estándares de medición e interpretación de información ${ }^{22}$. Estos datos recabados, además de ser instrumentos propios de la actividad gubernamental, también sirven para generar la imagen, en el colectivo social, de que se tiene control de la situación, que se conoce al fenómeno epidémico a fondo, así como el comportamiento de la enfermedad y, por lo tanto, generar una sensación de sosiego y certidumbre; es decir, de esperanza, la cual también es un instrumento del aparato biopolítico para combatir la llamada atmosfear (atmósfera del miedo) ${ }^{23}$.

En este sentido, el orden biopolítico mexicano difícilmente podrá cumplir con sus objetivos de preservar la vida humana de su población: 1) porque cuenta con un

\footnotetext{
${ }^{20}$ El gobierno mexicano, todos los días a las 19:00 horas informa desde el Palacio Nacional (sede del presidente de la República), a los medios de comunicación el estatus de la pandemia en el país. Para ello, invita especialmente a funcionarios públicos del sector salud.

${ }^{21}$ El presidente de la República, durante una gira de trabajo por el estado de Oaxaca los días 21 y 22 de marzo, invitó a la población a continuar con sus actividades cotidianas, especialmente las de índole económica, ya que el país todavía se encontraba en la fase 1 de la epidemia COVID-19. Al respecto, véase: Redacción, «Pese a coronavirus, pide AMLO "no dejar de salir" ni tomar medidas "exageradas"», El Universal, sección Nación, 22 de marzo, 2020, s/p., acceso: 01 de mayo, 2020,

https://www.eluniversal.com.mx/nacion/coronavirus-pide-amlo-no-dejar-de-salir-ni-tomarmedidas-exageradas

${ }^{22}$ Jorge Castillo Sepúlveda, «El Estado múltiple: el gobierno de las políticas basadas-en-laevidencia. Análisis desde los modos de individuación», en Iván, Pincheira Torres (et. al.) (eds) Máquinas del Saber, Mecanismos del Poder, Prácticas de Subjetivación (Chile: Ediciones Escaparate SPA, 2014), 91.

${ }^{23}$ Claes Tängh Wrangel, «Biopolitics of hope and security: governing the future through US counterterrorism communications», Globalizations, Vol. 16, n. 5 (2019): 664-665, acceso: 30 de abril, 2020, doi:10.1080/14747731.2018.1558631
}

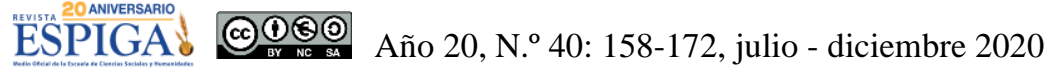


sistema de salud no suficientemente amplio con respecto al tamaño de la crisis sanitaria; 2) existe gran cantidad de ciudadanos con enfermedades crónicodegenerativas; 3) más de la mitad de la población en extrema pobreza y que, ante la profunda pauperización que conlleva la crisis económica producida por la pandemia, obligará a que no realicen la cuarentena, con ello están en riesgo de contagiar o ser contagiados, es decir, arriesgarse a morir por no tener para pagar los medios de subsistencia básica o por estar enfermos; de ahí, la dificultad para que se pueda extender y desarrollar una red biopolítica de protección y vigilancia para la población, por lo que el gobierno ha evitado la suspensión total de las actividades económico-comerciales y también ha propugnado por la pronta reactivación de las áreas estratégicas de la industria nacional. Por otra parte, tarde que temprano, solo pueden proteger a unos cuantos o quizás:

En este sentido, podemos ver que aunque la vida biológica y la de los sistemas políticos están profundamente interconectados, hay algo en la naturaleza de una exigencia, de un excedente objetivo de lo biológico sobre lo político, en la tensión impuesta por el virus en nuestros sistemas de salud, de distribución de alimentos y medicamentos, derechos laborales y las formas en que las oportunidades de vida se ven afectadas por distribuciones profundamente desiguales de riqueza e ingresos ${ }^{24}$ [traducción propia].

El orden biopolítico tiende a lo que Giorgio Agamben llamó el estado de excepción, donde la garantías jurídicas y el orden constitucional son suspendidos por el poder político bajo el argumento de salvaguardar la vida; es decir, se crean excepciones bajo argumentos legales que a todas luces no lo son ${ }^{25}$; así, por ejemplo, el Poder Ejecutivo el 23 de abril, mandó un proyecto de Decreto $^{26}$ a la Cámara de Diputados, con el fin de darle al presidente de la República (a través de la Secretaría de Hacienda y Crédito Público) poderes extraordinarios para disponer de los recursos públicos como mejor se considere bajo el argumento que se vive una situación de crisis y es indispensable esta medida, sin necesidad de que el poder legislativo (en particular, la cámara supra mencionada), lo apruebe o intervenga en la posible reasignación de recursos. Esta condición, si bien no pone a México a la altura de una dictadura, sí es una regresión autoritaria que poco abona a la transparencia y rendición de cuentas y al cumplimiento del orden constitucional.

Algunos estados de la República como Jalisco, Michoacán y Nuevo León, entre otros, en el afán de contener la epidemia, prácticamente han establecido gobiernos de excepción, donde la libre circulación está prohibida y hay bloqueos en caminos y entradas a ciudades, así como horarios acotados para estar en la vía pública y la

\footnotetext{
${ }^{24}$ Jonathan Short, «Biopolitical economies of the Covid-19 pandemic», Topia: Canadian Journal of Cultural Studies, Covid-19 Essays (2020): párr. 5, doi:10.3138/topia.2020.covid19.03, acceso: 28 de abril, 2020.

${ }^{25}$ Giorgo Agamben, Estado de Excepción. Homo Sacer II, I (Buenos Aires: Adriana Hidalgo Editora, 2005), 24.

${ }^{26}$ Para conocer el proyecto de decreto en su totalidad, así como la argumentación, véase: Presidencia de la Repúplica, «Iniciativa con proyecto de decreto por el que se adicionan diversas disposiciones de la Ley Federal de Presupuesto y Responsabilidad Hacendaria, remitida por el titular del Ejecutivo Federal», Gaceta Parlamentaria, año XXIII, n. ${ }^{\circ}$, 5506-I (27 de abril, 2020): 1-5, acceso: 29 de abril, 2020, http://gaceta.diputados.gob.mx/PDF/64/2020/abr/20200427-I.pdf

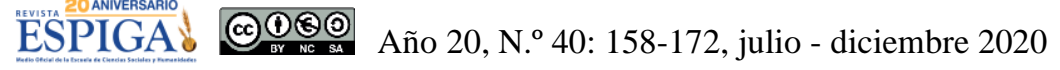


prohibición de venta de alcohol, como es el caso de Sinaloa. Estas medidas, además de ser inconstitucionales y violatorias de los derechos humanos, son contrarias a las políticas de autorregulación que ha promovido el gobierno federal, violaciones incluso reconocidas por la Secretaría de Gobernación (SG), quien:

(...) advirtió que la emergencia sanitaria por Covid-19 «no representa el establecimiento de un estado de excepción y suspensión de garantías». En un reporte mencionó a las entidades que han aplicado medidas «severas y desproporcionadas» al libre tránsito, las cuales, advirtió, no aseguran una reducción de los contagios. También informó que se han registrado «al menos 47 casos de agresiones» contra el personal de salud, con mayor rudeza hacia las enfermeras.

A partir de información de la Secretaría de Seguridad y Protección Ciudadana y otras fuentes, la SG indicó que en 340 municipios de 15 estados se ha restringido o controlado el acceso, ya sea en acciones promovidas por los alcaldes o la misma población ${ }^{27}$.

Existen ciertos sectores ciudadanos que en este momento actúan, junto con los gobernantes locales, de manera desproporcionada ${ }^{28}$, pero también bajo una lógica de preservar la vida a toda costa, de ahí que las tensiones y confrontaciones entre población y clase política se agudicen aún más. «Es ampliamente reconocido que el público no confía en los políticos. Lo que se discute con mucha menos frecuencia es que el problema de la confianza también funciona a la inversa. Las clases políticas no confían en el electorado, creen que los argumentos racionales y las apelaciones a la razón no tienen sentido y que es mucho mejor confiar en el giro y apelar a emociones como el miedo» [traducción propia] ${ }^{29}$, pero este estado de miedo, desconfianza y falta de transparencia solo hace que los efectos nocivos para las libertades y la vida humana se intensifiquen: «Así, en un círculo vicioso perverso, la limitación de la libertad impuesta por los gobiernos se acepta en nombre de un deseo de seguridad que ha sido impulsado por los propios gobiernos que ahora están interviniendo para satisfacerla ${ }^{30}$ [traducción propia].

\section{Conclusiones}

En el corto plazo, el Estado mexicano tiene que realizar cambios en el conjunto normativo vigente para que las epidemias, así como las enfermedades endémicas crónico-degenerativas (como diabetes, obesidad mórbida, entre otras), sean

\footnotetext{
${ }^{27}$ Fabiola Martínez, «SG: contraproducentes, los toques de queda y los retenes», La Jornada, miércoles 29 de abril, 2020: 6 .

${ }^{28}$ Se han reportado en diversas partes del país, pero especialmente en el estado de Jalisco, ataques al personal médico, bajo el pretexto de que pueden contagiar al resto de la población, estas agresiones van desde el hecho de no dejarlos usar el servicio de transporte público hasta agredirlos físicamente.

${ }^{29}$ Frank Furedi, «Fear and renunciation of politics», American Affairs II, n. 4 (2018): párr. 21, acceso: 30 de abril, 2020, https://americanaffairsjournal.org/2018/11/fear-and-therenunciation-of-politics/

${ }^{30}$ Giorgio Agamben, «L'invenzione di un'epidemia», Quodlbet (26 febbraio 2020): párr. 4, acceso: 30 de mayo, 2020, https://www.quodlibet.it/giorgio-agamben-l-invenzione-di-unepidemia
} 
consideradas amenazas a la seguridad nacional; para ello, es necesario reformas en los artículos 3 y 5 de la Ley de Seguridad Nacional, también debe reformarse el artículo 73, fracción XVI de la Constitución Política de los Estados Unidos Mexicanos para ampliar las funciones del Congreso en esta materia, así como dar mayores atribuciones al Consejo de Salubridad General. En este sentido, deberá modificarse la Ley de Planeación en su artículo 2, así como el Plan Nacional de Desarrollo, para que la noción de seguridad humana sea incluida en ambas normativas y, con ello, incidir en las políticas públicas, tanto de desarrollo social como económico, ajustándose también a los compromisos de los Objetivos del Desarrollo Sostenible y las metas específicas para el país; y, de esta manera, se pueda fortalecer todo el sistema de protección de la vida desde el nacimiento hasta la muerte, con ello también intentar dar mayor calidad y plenitud a la vida.

Los conceptos que Michel Foucault desarrolló en torno a la vida, el poder y las instituciones, como la gubernamentalidad, biopoder, biopolítica y poder soberano, son en este momento pertinentes, pues ayudan a explicar los dispositivos del poder y del gobierno sobre los vivos, que se desarrollaron desde el siglo XVIII y hasta la fecha. El aparato teórico que brinda el pensamiento biopolítico permite observar y descubrir cómo en las últimas tres décadas, los procesos de seguritización se han ampliado, así como las tendencias autoritarias, bajo el pretexto de proteger la vida siguen en uso de las élites políticas de buena parte del mundo y, la pandemia del COVID-19, es una circunstancia extraordinaria que permite ese discurso, pero también es la oportunidad para denunciar los abusos del biopoder.

El biopoder construyó aparatos y dispositivos de seguridad que pretenden mantener el control de la población, pero no por el control en sí mismo, sino para protegerla de agentes nocivos, tanto internos como externos, que pueden afectar la vida humana y, con ello, romper la armonía social. Las funciones de seguridad son las primeras que el Estado desarrolló en aras de un verdadero ejercicio del poder y no solo de carácter simbólico.

La gubernamentalidad política que el Estado mexicano ha emprendido para establecer dispositivos biopolíticos para la contención del COVID-19, así como la preservación de las instituciones, ha sido insuficiente debido al alto nivel de contagio que tiene esta enfermedad, además de la ineficacia institucional que no permite que se construya un frente en torno al combate eficaz de la pandemia. La vida humana es el punto central de cualquier gobierno (o debería serlo), es un poder sobre los vivos, para organizarlos de manera racional y, así, garantizar la continuidad del sistema del capital y la sociedad en su conjunto; pero, ante un escenario tan desalentador en México y en el mundo, es necesario replantear la manera de cómo se configuró el mundo desde hace siglos hasta el devenir actual; con ello, efectivamente proponer un gobierno que tenga no solo consideraciones biológicas y económicas, sino la plenitud y calidad de la existencia de todos los ciudadanos. 


\section{Formato de citación según APA}

Ortiz-Arellano, E. (2020). México ante el COVID-19: seguridad humana, gubernamentalidad y biopolítica. Revista Espiga, 20 (40), páginas 158-172.

\section{Formato de citación según Chicago-Deusto}

Ortiz-Arellano, Edgar. «México ante el COVID-19: seguridad humana, gubernamentalidad y biopolítica». Revista Espiga 20, n. ${ }^{\circ} 40$ (julio-diciembre, 2020): páginas 158-172.

\section{Fuentes consultadas}

Agamben, Giorgio. «L'invenzione di un'epidemia». Quodlbet (26 febbraio 2020). Acceso: 30 de mayo, 2020. https://www.quodlibet.it/giorgio-agamben-1invenzione-di-un-epidemia

Agamben, Giorgio. Estado de Excepción. Homo Sacer II, I. Buenos Aires: Adriana Hidalgo Editora, 2005.

Castillo Sepúlveda, Jorge. «El Estado múltiple: el gobierno de las políticas basadasen-la-evidencia. Análisis desde los modos de individuación», en Pincheira Torres, Iván. (et. al.) (eds) Máquinas del Saber, Mecanismos del Poder, Prácticas de Subjetivación. Chile: Ediciones Escaparate SPA, 2014, 89-96.

Castro, Edgardo. Diccionario Foucault. Temas, Conceptos y Autores. Buenos Aires: Siglo XXI Editores, 2011.

Constitución Política de los Estados Unidos Mexicanos (Ciudad de México: Diario Oficial de la Federación, última reforma 06-03-2020). Acceso: 27 de abril, 2020. http://www.diputados.gob.mx/LeyesBiblio/pdf/1_060320.pdf

Davitti, Daria. «Biopolitical borders and the state of exception in the european migration "crisis" ». European Journal of International Law 29, n. 4 (2018): 1173-1196. Acceso: 25 de abril, 2020. https://doi.org/10.1093/ejil/chy065

Dillon, Michael and Luis, Lobo-Guerrero. «Biopolitics of security in the 21st century: An introduction». Review of International Studies 34, n. 2 (2008): 265-292. Acceso: 30 de julio 2020. En DOI: 10.1017/S0260210508008024

Duffield, Mark. «Seguridad humana: vincular desarrollo y seguridad en una era de terror». Relaciones Internacionales, n. 43 (2020): 11-32. Acceso: 25 de abril, 2020.

https://revistas.uam.es/index.php/relacionesinternacionales/article/view/rel acionesinternacionales2020.43.001/11880

Estévez, Ariadna. «La repolitización de los derechos humanos frente a la gubernamentalidad neoliberal del sufrimiento social: una lucha de

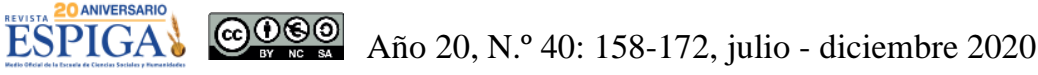


contraconducta», en Estévez, Ariadna y Daniel Vázquez (coords.) 9 Razones para (Des)confiar de las Luchas por los Derechos Humanos. Ciudad de México: Universidad Nacional Autónoma de México, Facultad Latinoamérica de Ciencias Sociales, Sede México, 2017, 181-208.

Foucault, Michel. Defender la Sociedad. Curso en el Collège de France (19751976). Ciudad de México: Fondo de Cultura Económica, 2006.

Foucault, Michel. El Poder, una Bestia Magnífica. Ciudad de México: Siglo XXI Editores, 2013.

Foucault, Michel. Seguridad, Territorio y Población. Curso en el Collège de France (1977-1978). Ciudad de México: Fondo de Cultura Económica, 2006.

Furedi, Frank. «Fear and renunciation of politics». American Affairs, Vol. II, n. ${ }^{\circ} 4$ (2018). Acceso: 30 de abril, 2020.

https://americanaffairsjournal.org/2018/11/fear-and-the-renunciation-ofpolitics/

Gobierno de México. Comunicado Técnico Diario COVID-19 México. Ciudad de México: Gobierno de México. Acceso: 30 de julio, 2020. https://www.gob.mx/cms/uploads/attachment/file/566650/Comunicado_Te cnico_Diario_COVID-19_2020.07.29.pdf

Hardt, Michael y Antonio Negri. Imperio. Buenos Aires: Paidós, 2002.

Hernández García, Nuria. «La seguridad humana: del concepto al enfoque. Causas de la reducción de su uso como concepto». Relaciones Internacionales, $\mathrm{n}^{\circ}$ 43 (2020): 33-48. Acceso: 31 de julio, 2020.

https://doi.org/10.15366/relacionesinternacionales2020.43.002

Jaeger, Hans-Martin. «UN reform, biopolitics, and global governmentality». International Theory II, Issue 1 (2010): 50-86. Acceso: 01 de mayo, 2020. DOI: $10.1017 / \mathrm{s} 1752971909990182$

Ley de Seguridad Nacional. Ciudad de México: Diario Oficial de la Federación, últimas reformas 08-11-2019. Acceso: 27 de abril, 2020. http://www.diputados.gob.mx/LeyesBiblio/pdf/LSegNac_081119.pdf

Ley General de Salud. Ciudad de México: Diario Oficial de la Federación, últimas reformas 24-01-2020. Acceso: 27 de abril, 2020.

http://www.diputados.gob.mx/LeyesBiblio/pdf/142_240120.pdf

Martínez, Fabiola. «SG: contraproducentes, los toques de queda y los retenes». La Jornada, miércoles 29 de abril, 2020.

Periago, Mirta Roses. «Seguridad humana y salud pública: [editorial]». Revista Panamericana de Salud Pública 31, n. 5 (2012): 351-354. Acceso: 26 de abril, 2020.

https://iris.paho.org/bitstream/handle/10665.2/9332/v31n5a00b_351-

4.pdf? sequence $=1 \&$ isAllowed $=\mathrm{y}$

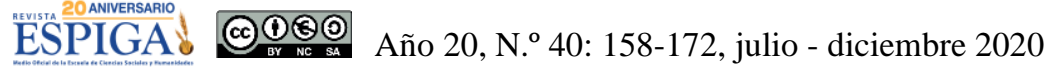


Presidencia de la Republica. «Iniciativa con proyecto de decreto por el que se adicionan diversas disposiciones de la Ley Federal de Presupuesto y Responsabilidad Hacendaria, remitida por el titular del Ejecutivo Federal». Gaceta Parlamentaria, Año XXIII, n. ${ }^{\circ}$ 5506-I (27 de abril, 2020). Acceso: 29 de abril, 2020.

http://gaceta.diputados.gob.mx/PDF/64/2020/abr/20200427-I.pdf

Redacción. «Pese a coronavirus, pide AMLO "no dejar de salir" ni tomar medidas “exageradas"». El Universal, sección Nación, 22 de marzo de 2020. Acceso: 01 de mayo, 2020. https://www.eluniversal.com.mx/nacion/coronaviruspide-amlo-no-dejar-de-salir-ni-tomar-medidas-exageradas

Short, Jonathan. «Biopolitical economies of the Covid-19 pandemic». Topia: Canadian Journal of Cultural Studies. Covid-19 Essays (2020). Acceso: 28 de abril, 2020. DOI:10.3138/topia.2020.covid-19.03

Wrangel, Claes Tängh. «Biopolitics of hope and security: governing the future through US counterterrorism communications». Globalizations 16 , n. ${ }^{\circ} 5$ (2019): 664-677. Acceso: 30 de abril, 2020.

DOI:10.1080/14747731.2018.1558631 